\title{
On radio source selection to define a stable celestial frame ${ }^{\star}$
}

\author{
S. B. Lambert and A.-M. Gontier
}

\begin{abstract}
Observatoire de Paris, Département Systèmes de Référence Temps Espace (SYRTE), CNRS/UMR8630, 75014 Paris, France
e-mail: [sebastien.lambert; anne-marie.gontier]@obspm.fr
\end{abstract}

Received 12 July 2008 / Accepted 7 October 2008

\begin{abstract}
Context. The most accurate realization of a quasi inertial reference frame, the International Celestial Reference Frame (ICRF), is made of 212 defining extragalactic radio sources whose coordinates are determined using VLBI observations. Recent studies demonstrated however that using other sets of sources could improve the frame stability.

Aims. This study examines a simple radio source selection scheme to define celestial reference frame axes more stable than the ones as currently defined by the ICRF.

Methods. After having derived source coordinate time series from 26 years of VLBI observations, we select the most suitable sources on the basis of their positional variability (rms and slope), and observational history. We determine the axis stability of the frame defined by the selection, as well as its suitability for global geodetic VLBI analyses, i.e., determination of Earth orientation parameters and source and station coordinates.

Results. We select four frames made of 196, 200, 262, and 269 sources, respectively, showing a satisfactory sky coverage in both hemispheres. Our selections provide a frame stability improved by up to $40 \%$ with respect to the ICRF, and by $20 \%$ with respect to the frame as defined by the 247 stable sources of Feissel-Vernier et al. (2006, A\&A, 452, 1107). Reanalysis of data with respect to this frame gives astrometric catalogues aligned to the ICRF-Ext. 2 within $17 \mu$ as. Effects on the Earth orientation parameter estimates and terrestrial reference frame determination remain marginal.

Conclusions. In view of the generation of the next ICRF, we recommend that such an algorithm be taken into consideration along with other criteria based on individual characteristics of the radio sources.
\end{abstract}

Key words. reference systems - Earth

\section{Introduction}

Precise astrometry and geodesy need the definition of the quasi inertial reference frame to which observations can be tied. By the mid-1990s, thanks to the very long baseline interferometry (VLBI) technique, such a frame was defined by the radio positions of 212 extragalactic sources, also known as "defining sources" of the International Celestial Reference Frame (ICRF; Ma et al. 1998) which was adopted by the International Astronomical Union (IAU) in 1995 as primary realization of the International Celestial Reference System. These sources have a positional accuracy better than 1 milliarc second (mas) in both coordinates. They have been used for more than ten years for geodetic VLBI data reduction (to obtain, e.g., Earth orientation parameters (EOP) or radio source catalogues) in order to tie the observations to a reference frame that is supposedly globally non rotating with respect to the far universe.

Nevertheless, several recent studies by Feissel-Vernier (2003), and Feissel-Vernier et al. (2006) have shown that the positional stability of the 212 defining sources of the ICRF is questionable. The authors proposed other ensembles of sources that could provide more reliable reference frames. In the latter study, the authors provided a sample of 247 sources that were selected for their high positional time stability. The reference frame

* The source list of the AMS subsets is only available in electronic form at the CDS via anonymous ftp to cdsarc.u-strasbg. fr $(130.79 .128 .5)$ or via

http://cdsweb.u-strasbg.fr/cgi-bin/qcat?J/A+A/493/317 realized with this sample was shown to be substantially more stable than the 212 ICRF defining sources.

Other studies have been carried out through the geodetic VLBI community, mainly to assess the astrometric quality of the Feissel-Vernier et al. selected sources after time series of astrometric positions, and to evaluate the effects of the source positional instabilities on various VLBI-derived geodetic parameters like Earth orientation parameters, station positions, or geophysical parameters describing the Earth's interior (Titov 2007; Sokolova 2008; Lambert et al. 2008). It emerges from these studies that there exists some inconsistencies between the different sets, especially when the stability indices of Feissel-Vernier et al. are compared to the structure indices computed using Very Long Baseline Array observations by Fey \& Charlot (2000). It also appears that the analysis strategy to handle sources that have a highly unstable position, in order to minimize the pollution of geodetic parameters, has to be investigated. Indeed, effects of estimating or not estimating coordinates of an unstable source can amount to several tens of microarc seconds in the EOP (MacMillan \& Ma 2007; Sokolova 2008).

It appears that all these studies investigated existing sets of defining sources in terms of individual source positional stability, but none of them tried to measure the frame stability nor to search for a new set of sources able to replace the existing ones. An attempt in this direction was proposed by Gontier \& Lambert (2008) where the authors selected about 200 sources and concluded that the axes of the reference frame as defined by these sources' coordinates were more stable than the current 
ICRF axes. At that time, the study did not investigate further the ability of the selection to improve VLBI products.

The current effort in view of the second realization of the ICRF (to be approved at the IAU 2009 General Assembly) addresses one more time the problem of the choice of the defining sources: a source sample able to define stable axes for future astrometric and geodetic VLBI data reduction and reanalysis of past observations. Determination of new subsets with improved stability constitutes the purpose of this paper. In the following two sections, radio source coordinate time series are used to select ensembles of sources that define stable reference frame axes. In the next section, we use our selected ensembles as reference sources in global VLBI solutions to examine the effects on astrometric catalogues, EOP and terrestrial reference frame.

For clarity, the 212 ICRF defining sources will be referred to as "212 ICRF" in the following. The 247 stable sources of Feissel-Vernier et al. will be further named "247 MFV". Since the work of Feissel-Vernier (2003), the term "stability" has extensively been used to qualify the suitability of a source to be part of an ensemble that would define a stable reference frame over the observing time span. In that sense, it is wise to give the sources a "stability index" that describes the level of astrometric positional instability as measured by VLBI. However, the stability index should not be interpreted as an intrinsic characteristic of the source, but rather as an astrometric qualifier that obviously strongly depends on the intrinsic evolution of the source structure and flux but also, for a significant part, on the VLBI observing strategy. We are reticent to employ the words "stable" or "unstable" when refering to a source: there is a risk that these terms could be interpreted as two separate categories of extragalactic objects. Since it has been observed that most of the sources can appear to be stable for a long time and, suddenly, show a violent shift of the radio center, such a classification seems astrophysically meaningless. Such a discussion would need more astrophysical considerations about extragalactic objects and is out of the scope of this paper. In our paper, we will employ the word "suitable" with regards to the sources having a stable astrometric position. The word "stable" will rather be employed when speaking about the reference frame.

\section{Source selection and frame stability}

\subsection{Generation of source coordinate time series}

Coordinate time series of 655 sources are obtained by the analysis of 3800 diurnal sessions involving more than two baselines between January 1984 and March 2008. The full set of time series is obtained after six solutions. In each solution, one sixth of the sources' coordinates are estimated as session parameters. Others are estimated as global parameters, including five sixth of the $247 \mathrm{MFV}$ that are constrained by a no-net rotation (NNR) condition with a priori coordinates taken in the ICRF-Ext. 2 catalogue (Fey et al. 2004). In all solutions, we set the elevation cut-off at $5^{\circ}$. Station coordinates are estimated as session parameters with no constraint. An a priori zenith delay is given by the Niell (1996) mapping functions. Zenith delay and gradients are estimated every $20 \mathrm{mn}$ and $6 \mathrm{~h}$, respectively. Analyses are done using the Calc 10.0/Solve 2006.06.08 geodetic VLBI analysis software package and are led from the Paris observatory IVS analysis center (OPAR), part of the International VLBI Service for Geodesy and Astrometry (IVS; Schlüter \& Behrend 2007).

At each session, the set of observed source coordinates constitutes a local reference frame. To check whether the series of local frames remain undeformed with time (in other terms,

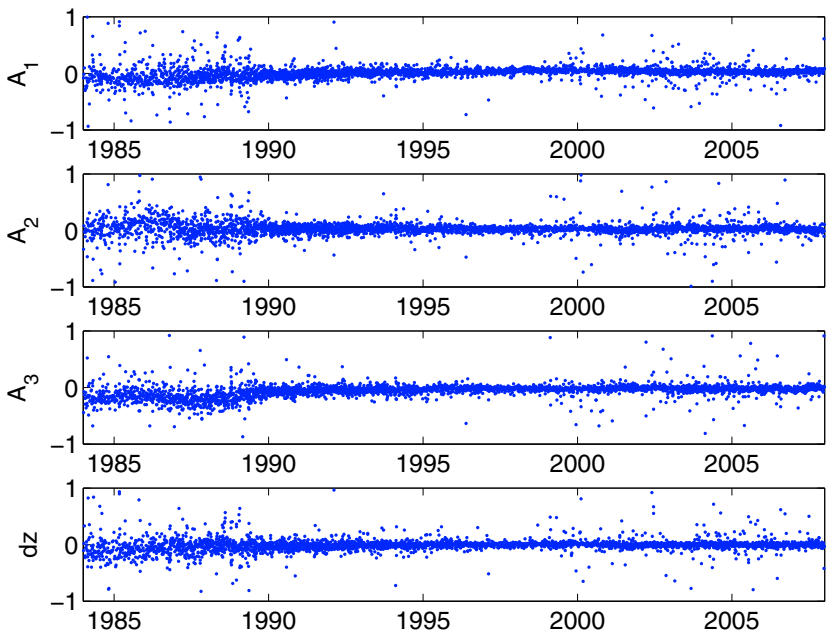

Fig. 1. Deformability parameters of local reference frames as realized with the coordinate time series used in this paper. The units are mas.

whether the NNR condition is efficient), we compute local transformation parameters between local frames and the a priori frame as given by the ICRF-Ext. 2 source coordinates. The coordinate difference is modeled by a 4-parameter transformation including three rotations of angles $A_{1}, A_{2}$ and $A_{3}$, around the $X$, $Y$ and $Z$ axes of the celestial frame, respectively, and a parameter $d z$ accounting for a global translation of the source coordinates in declination (see, e.g., IERS 1996, or Feissel-Vernier et al. 2006):

$\Delta \alpha=A_{1} \tan \delta \cos \alpha+A_{2} \tan \delta \sin \alpha-A_{3}$,

$\Delta \delta=-A_{1} \sin \alpha+A_{2} \cos \alpha+d z$.

This transformation is a simplified form of a more sophitiscated transformation based on a spherical harmonics decomposition of the vector field of coordinate differences (Mignard \& Morando 1990). Other interesting transformations based on Legendre-Fourier functions have been developed by Sokolova \& Malkin (2007) to model differences between catalogues of several hundreds of sources. Here, we only keep degree 1 and 2 describing translations and rotations, respectively. Higher degrees would be irrelevant since we only treat a small number of sources. Local values of the four parameters are reported in Fig. 1. The typical formal error is 0.2 mas. The flatness of the curve after 1990 testifies that the solution is reasonably undeformed. Before 1990, the general deficiency of the VLBI networks, including the number of observed sources and observing antennas per session, likely explains the observed departures of the angles (see, e.g. Gontier et al. 2001; Malkin 2004; FeisselVernier et al. 2004, who report interesting statistical results and remarks about the VLBI evolution over the last two decades). For this reason we will base our following analyses only on data after 1990.

From session coordinate time series, we compute semiannual points by applying a weighted moving average. The averaging window is of length 2 years and is displaced by steps of 0.5 year. The semi-annual point within the window is obtained by the weighted average of the contained source coordinates when the number of sessions is at least 3. Otherwise, the point is set to zero together with a very high uncertainty. Doing so, we assume that a half year is representative of the time scale on which a significant radio center displacement can occur. To exclude sources having a poor observational history and for which the characterization of the behavior would be questionable, we 
only keep time series for sources delivering at least ten semiannual points, i.e., 433 sources.

\subsection{Selection of suitable sources}

We then apply a selection scheme to detect sources that will be able to define a stable reference frame. In that context, stable means that the axis direction will remain within a limited area over the entire observational time span. To isolate suitable sources, we consider the root mean square (rms) and the slope of both the session time series and their semi-annual counterparts, as well as the length (or number of points) of the semi-annual point time series. This algorithm is similar in some points to the ones used in the various studies initiated by Feissel-Vernier in the past years, although she and her collaborators used additional estimators such as the goodness of fit or the Allan variance (see Feissel \& Gontier 2000; Gontier et al. 2001, and the already cited papers of Feissel-Vernier).

We consider the rms of both the session and the semi-annual point time series since they describe different aspects of the radio center motion. Indeed, a high rms in the semi-annual point time series generally means a violent underlying physical phenomenon (e.g., plasma jets), while a high rms in the session time series can simply reflect a random-like behavior of the apparent radio center due to, e.g., network effects.

We do not physically interprete the slope of a coordinate time series. Instead, we assume that it is the signature of any variation occuring on a time scale that is much longer than the observational time span. A significant slope obviously leads to an immediate rejection of the source. However, if the slope is not extreme, its significance is measured by its ratio to its uncertainty. A quite large slope associated with a large uncertainty means it is non significant. In constrast, a source coordinate time series showing a slope with a small uncertainty has to be considered with great care. If the source motion shows a slope higher than $0.1 \mathrm{mas} / \mathrm{yr}$ or a ratio of the slope to its uncertainty larger than 15 in session-wise time series and 5 in semi-annual point time series, it is considered as non suitable for our purpose. To be retained by our algorithm, a source must pass the test successfully for both session-wise and semi-annual point time series. These levels of significance have been fixed after several tests in order to obtain a reasonable number of selected sources. Too tight constraints lead to subsets too small to define a reliable frame of reference.

An additional parameter in our selection scheme is the length of the time series. We tune this parameter to assess the evolution of the frame stability when the number of selected sources constituting this frame increases. Semi-annual reference frames, realized by the semi-annual coordinates of the selected radio sources if observed at the considered epoch, are compared to the same sources' coordinates as given in the ICRF-Ext.2. The average axis stability of the frame is then computed as the rms of the time variability of the transformation parameters. Figure 2 displays the axis stability for various frames made of an increasing number of sources (thick line). The horizontal line pairs represent the values relevant to the frames defined by the 212 ICRF defining sources (dashed line), and by the 247 stable sources of Feissel-Vernier et al. (dotted line), respectively. It appears that our selections lead to substantially better stabilities for $A_{1}, A_{3}$, and $d z$ compared to $212 \mathrm{ICRF}$ and $247 \mathrm{MFV}$. However, for $A_{2}$, the stability is comparable to 212 ICRF, and worse than $247 \mathrm{MFV}$. The bottom plot represents the average of the four transformation parameters: the stability stays around $21 \mu$ as which is slightly below $247 \mathrm{MFV}$.
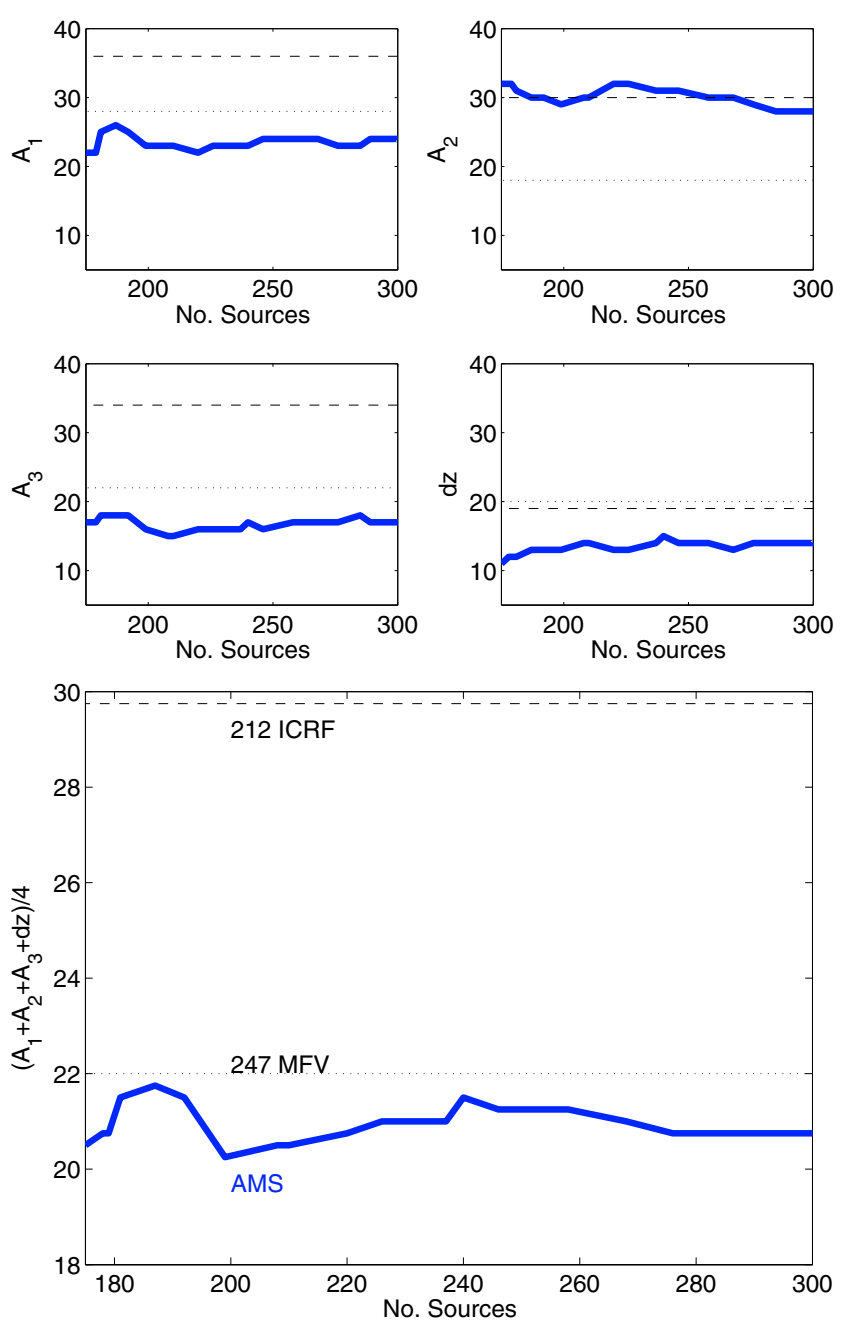

Fig. 2. Axis stability of the celestial frame versus the number of selected sources (see details in the text). The units are $\mu$ as.

\subsection{Construction of reference frames}

Now, what we are seeking to determine is a subset of defining sources. Following Fig. 2, any subset, chosen without refinement within the figure, would lead to improving stabilities for $A_{1}, A_{3}$, and $d z$. For $A_{2}$, the stability would be at worse slightly higher than 212 ICRF.

For illustration purpose, we select two subsets that will be studied in more detail. One subset will have a relatively small number of sources, fewer than the 212 defining sources of the ICRF. The other subset will have a large number, comparable to the 247 sources of Feissel-Vernier et al. (2006). The first subset contains 200 sources and will be further referred to as "200 AMS". 71 sources are part of the 212 ICRF and 135 are part of the $247 \mathrm{MFV}$. The second subset contains 269 sources (referred to as "269 AMS") of which 116 are part of the 212 ICRF and 171 are in the $247 \mathrm{MFV}$. The above results mean that about $30 \%$ of the $247 \mathrm{MFV}$ sources were not selected by our algorithm. Causes for this discrepancy arise from the semi-annual point computation, the different selection algorithm, and the extra four years of data. The stabilities of the various frames are reported in Table 1. Except for $A_{2}$, the AMS sources lead to a stability at the level or better than the $247 \mathrm{MFV}$. The AMS sources also lead to a significantly better stability than the 212 ICRF. 
Table 1. Axis stability of the celestial frames investigated in this study. The units are $\mu$ as.

\begin{tabular}{rrrrrrr}
\hline \hline & 212 & 247 & 200 & 269 & 196 & 262 \\
& ICRF & MFV & AMS & AMS & AMS & AMS \\
\hline$A_{1}$ & 36 & 28 & 23 & 24 & 21 & 22 \\
$A_{2}$ & 30 & 18 & 29 & 30 & 18 & 21 \\
$A_{3}$ & 34 & 22 & 16 & 17 & 16 & 17 \\
$d z$ & 19 & 20 & 13 & 13 & 13 & 13 \\
\hline
\end{tabular}

It appears that the stability of $A_{2}$ is degraded with respect to $247 \mathrm{MFV}$ in both the AMS 200 and AMS 269 selections. It is likely that the sources responsible for the discrepancy of AMS 269 are those which are in 269 AMS and not in $247 \mathrm{MFV}$, let 98 sources with a positional weighted rms ranging from 0.2 mas up to 2.5 mas. Only a dozen among them show a rms higher than 1 mas. By progressively removing the most unstable of these sources from 269 AMS, we build a reference frame of 262 sources ("262 AMS") whose stability is reported in Table 1. The stability in $A_{3}$ and $d z$ is the same as in 269 AMS. The stability in $A_{1}$ is slightly improved. The stability in $A_{2}$ is now at the level of $247 \mathrm{MFV}$, although slightly higher. The same treatment applied to AMS 200 leads to the "AMS 196" frame that contains 196 sources, and that provides the best stability of all the frames studied in this paper.

Figure 3 reports on the 98 extra sources that are in 269 AMS and not in $247 \mathrm{MFV}$. The envelopes of variability are obtained for each source by computing the weighted rms in a given direction. These 98 sources appear to be mostly in the southern hemisphere. The lower plots display a better view of the envelopes of variability and the semi-annual point time series of the sources that were removed from 269 AMS to arrive at the final 262 AMS selection. The reason why Feissel-Vernier et al. ignored these sources in $247 \mathrm{MFV}$ is likely that their observation starts later than 1995. Since Feissel-Vernier et al. rejected sources with too short an observational history, these were rejected. However, the extra four years of data in our study enabled us to select them. One can also notice that most of these sources show extended structures $^{1}$ with corresponding structure indices higher than 3 (Fey \& Charlot 2000).

Source list of the AMS subsets are made available along with this paper in electronic form.

The sky distribution of the sources is displayed in Fig. 4. The coverage of the southern hemisphere is more complete for the $247 \mathrm{MFV}$ and the AMS sources than for the ICRF sources due to the densification programs undertaken after the construction of the ICRF (relevant information concerning VLBI CRF sessions dedicated to densifying the southern hemisphere can be found in Fey et al. 2004). The mean declination of the subsets are $15^{\circ}$ for $212 \mathrm{ICRF}, 17^{\circ}$ for $247 \mathrm{MFV}$, and $8^{\circ}$ for the AMS selections, indicating that our subsets are more closely centered on the equator. Figure 4 also shows the envelopes of variability of the 655 sources, with the 262 AMS sources drawn in red. It appears that the AMS sources have smaller envelopes, and that the size of the envelopes increases as the declination decreases from $90^{\circ} \mathrm{N}$ to $90^{\circ} \mathrm{S}$ (see Fig. 5). This fact, already pointed out in FeisselVernier et al. (2006), possibly results from a miscorrected troposphere delay and still has to be investigated and fixed.

1 The Radio Reference Frame Image Database provides VLBA maps at $8.4 \mathrm{GHz}$. This service is hosted at the Astrometry Department of the United States Naval Observatory, Washington, DC (http: //rorf. usno . navy . mil/RRFID).
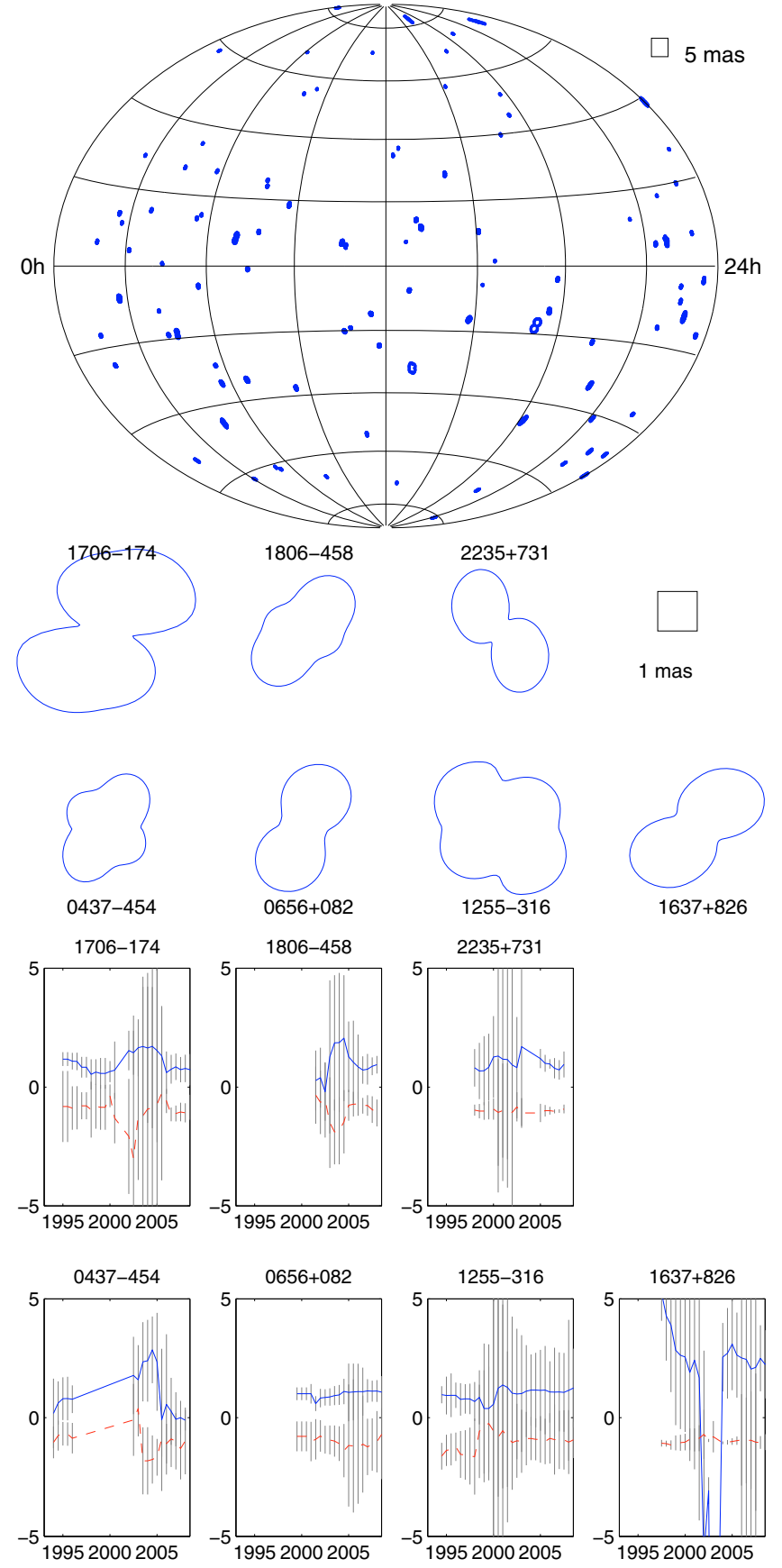

Fig. 3. Distribution of the 98 extra sources that are in 269 AMS and not in $247 \mathrm{MFV}$ (top), and envelopes of variability (middle) and semiannual point time series representation of the sources that were removed from 269 AMS to arrive at the final 262 AMS selection (bottom). For the time series, the blue, solid line is $\alpha \cos \delta$ shifted by 1 mas; the red, dashed line is $\delta$ shifted by -1 mas. The mean values have been removed. The units are mas.

\section{Effect on astrometric and geodetic VLBI results}

To test the efficiency of the AMS subsets, we produce catalogues and Earth orientation parameter time series. The analysis strategy we use at the OPAR IVS analysis center is very close to the strategies in use in other IVS analysis centers, so that we expect that the results obtained here are still relevant. All sessions already used for time series generation are analyzed. Coordinates of 655 sources are set as global parameters. Coordinates of 277 sources observed in less than 20 sessions are estimated as 

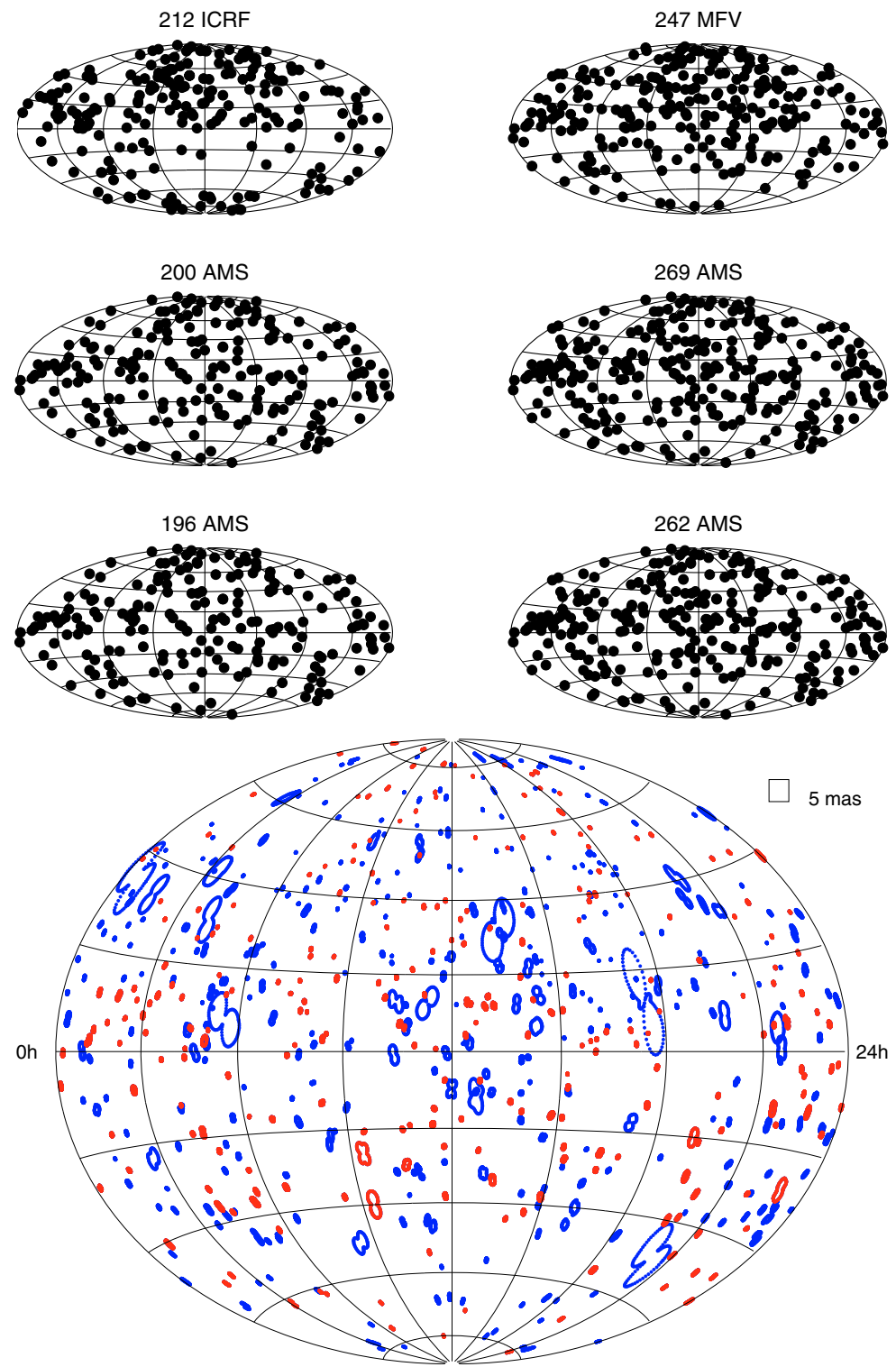

Fig. 4. Distribution of the source subsets considered in this work (top), and envelopes of variability of the 655 observed sources (blue) and of the final selection 262 AMS sources (red) (bottom).

session parameters. All but two stations' coordinates and velocities are estimated as global parameters. Station HRAS 085, located at Fort Davis, Texas, is modeled by B-spline functions of degree 3 over 15 nodes between 1980 and 1992. The non linear motion of Fairbanks, Alaska, caused by the post-seismic transient after the 2002 Denali fault earthquake (MacMillan \& Cohen 2004; Titov \& Tregoning 2004, 2005) is also modeled by B-spline functions (Petrov 2005) of degree 2 over 9 nodes between 1984 and 2006. The source coordinates are constrained by a NNR with respect to each of the studied subsets (i.e., successively 212 ICRF, $247 \mathrm{MFV}$, and the AMS subsets, that further will be referred to as reference radio sources). Polar motion and UT1 are estimated as session parameters together with nutation offsets and length-of-day. Coordinates and velocities of 35 stations are constrained by no-net rotation and no-net translation with respect to the ITRF 2000.

The obtained radio source coordinate catalogues are named after the reference radio source subset and are then compared to the ICRF-Ext.2. An examination of the differences source by source reveals that the determination of source positions can change by about $100 \mu$ as from one catalogue to another. This feature is mainly the result of global rotation between them. Table 2 displays some statistics. Comparison of the formal errors are made for sources having more than 1000 observations. We note improvements of $\sim 30 \%$ for $\alpha \cos \delta$ and $\sim 20 \%$ for $\delta$ for the $247 \mathrm{MFV}$ and AMS solutions with respect to the 212 ICRF. The transformation parameters adjusted to the coordinate differences for 587 common sources are reported in Table 2. It appears that applying the NNR on the AMS sources leads to significantly smaller biases against the ICRF-Ext.2. The angles for the AMS solutions appears to be non significant (at the $1 \sigma$ level), whereas both the 212 ICRF and the 247 MFV catalogues show significant departures in $A_{1}$ and $A_{2}$. Once the source coordinates are corrected using Table 2 parameters, the remaining differences between catalogues never exceed $1 \mu$ as.

Differences in nutation offset time series are addressed in Table 3 for the bias, slope and quadratic term. The bias can change drastically from one solution to another, by an amount comparable to the corresponding rotation in Table 2. (A rotation of $A_{1}$ around the $X$-axis leads to a corresponding bias in $d Y$. 
Table 2. Statistics of the positions and standard errors, and transformation parameters of the astrometric catalogues produced in this study against the ICRF-Ext.2. The units are $\mu$ as.

\begin{tabular}{|c|c|c|c|c|c|c|c|c|}
\hline & & 212 ICRF & $247 \mathrm{MFV}$ & 200 AMS & 269 AMS & 196 AMS & 262 AMS & Formal error \\
\hline \multirow[t]{2}{*}{ Average positional difference } & $\alpha \cos \delta$ & 15 & 11 & 8 & 8 & 6 & 6 & \\
\hline & $\delta$ & 12 & 13 & 16 & 15 & 15 & 15 & \\
\hline \multirow[t]{2}{*}{ Rms of the positional difference } & $\alpha \cos \delta$ & 216 & 218 & 218 & 218 & 218 & 218 & \\
\hline & $\delta$ & 230 & 223 & 223 & 223 & 222 & 222 & \\
\hline \multirow{2}{*}{ Average formal error } & $\alpha \cos \delta$ & 119 & 118 & 118 & 118 & 118 & 118 & \\
\hline & $\delta$ & 137 & 137 & 137 & 137 & 137 & 137 & \\
\hline$\overline{A_{1}}$ & & -51 & 25 & 17 & -2 & -15 & 3 & \pm 17 \\
\hline$A_{2}$ & & 30 & 0 & -16 & -16 & -15 & -18 & \pm 16 \\
\hline$A_{3}$ & & -13 & -13 & -6 & -8 & -9 & -6 & \pm 16 \\
\hline$d z$ & & 14 & 14 & 14 & 14 & 14 & 14 & \pm 16 \\
\hline
\end{tabular}

Table 3. Second-order polynomial coefficients adjusted in nutation offset time series obtained from the solutions considered in this study. Values are against the IAU 2000A nutation model of Mathews et al. (2002). Coefficients of $t^{0}$ are in mas, of $t^{1}$ in mas/cy, and of $t^{2}$ in mas/cy².

\begin{tabular}{rrrrrrrrrrrrrr}
\hline \hline & \multicolumn{2}{c}{ 212 ICRF } & \multicolumn{2}{c}{247 MFV } & \multicolumn{2}{c}{ 200 AMS } & \multicolumn{2}{c}{ 269 AMS } & \multicolumn{2}{c}{ 196 AMS } & \multicolumn{2}{c}{ 262 AMS } \\
& \multicolumn{1}{c}{$d X$} & $d Y$ & $d X$ & $d Y$ & $d X$ & $d Y$ & $d X$ & $d Y$ & $d X$ & $d Y$ & $d X$ & $d Y$ \\
\hline$t^{0}$ & 0.018 & -0.084 & 0.049 & -0.008 & 0.065 & -0.050 & 0.064 & -0.035 & 0.064 & -0.048 & 0.066 & -0.030 \\
& \pm 0.002 & \pm 0.002 & \pm 0.002 & \pm 0.002 & \pm 0.002 & \pm 0.002 & \pm 0.002 & \pm 0.002 & \pm 0.002 & \pm 0.002 & \pm 0.002 & \pm 0.002 \\
$t^{1}$ & 0.817 & -1.315 & 0.811 & -1.316 & 0.812 & -1.314 & 0.812 & -1.311 & 0.812 & -1.313 & 0.812 & -1.311 \\
& \pm 0.025 & \pm 0.025 & \pm 0.024 & \pm 0.024 & \pm 0.024 & \pm 0.024 & \pm 0.024 & \pm 0.024 & \pm 0.024 & \pm 0.024 & \pm 0.024 & \pm 0.024 \\
$t^{2}$ & 6.227 & -12.506 & 6.064 & -12.497 & 6.078 & -12.462 & 6.076 & -12.429 & 6.083 & -12.459 & 6.081 & -12.434 \\
& \pm 0.434 & \pm 0.427 & \pm 0.427 & \pm 0.419 & \pm 0.427 & \pm 0.419 & \pm 0.428 & \pm 0.420 & \pm 0.427 & \pm 0.419 & \pm 0.427 & \pm 0.420 \\
\hline
\end{tabular}
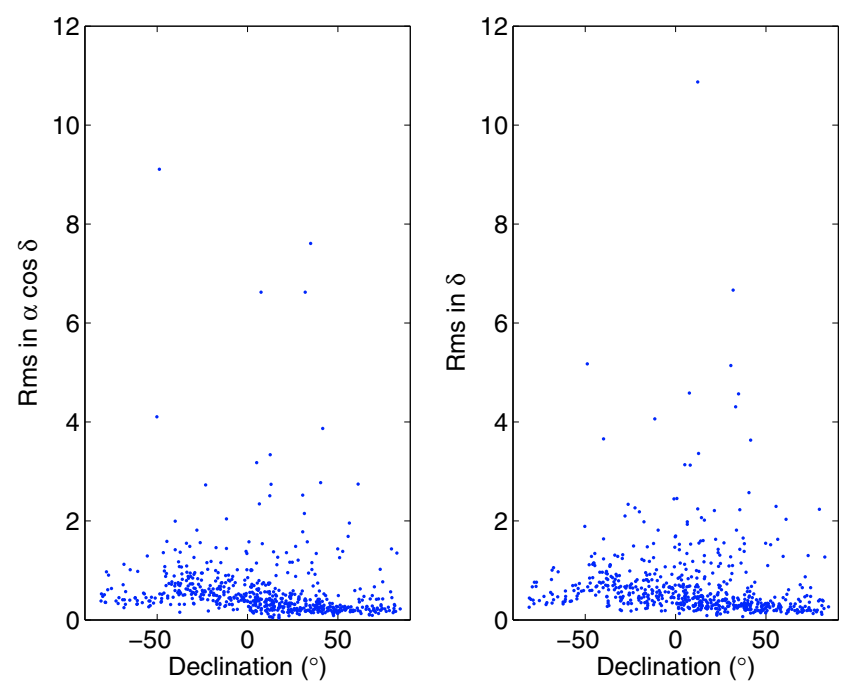

Fig. 5. Rms in right ascension and declination for the 655 observed sources as a function of the declination. The units are mas.

Similarly, a rotation of $A_{2}$ around the $Y$-axis produces a bias in $d X$.) These differences, that can reach more than $100 \mu$ as, can therefore be explained by reference frame effects. Higher orders are far less affected. The amplitude of tidal terms (e.g., 18.6-yr) are also modified very marginally. No implication is expected for further determination of geophysical parameters (e.g., dynamical flattening and frequencies associated with the rotational normal modes of the internal layers, and that can be deduced from the comparison of observed nutation amplitudes at tidal frequencies with the corresponding amplitudes as predicted by the theory).

Feissel-Vernier et al. (2006) presented comparisons between VLBI solutions where the NNR was applied successively on the 212 ICRF with all source coordinates estimated as global parameters (solutions tagged $c n 7$ and $c n 8$ in their paper) and on the $247 \mathrm{MFV}$ with selected unstable source coordinates downgraded to local parameters (cne and $c n h$ ). They noticed significant discrepancies between the EOP estimates coming from their various solutions. MacMillan \& Ma (2007), Titov (2007) and Lambert et al. (2008) investigated the effects of the selection of reference radio sources in geodetic products. To achieve this, they produced several VLBI solutions using various strategies in which the reference radio sources as well as the sources whose coordinates are estimated as global/local parameters were different. All these works showed that significant variations in EOP originate in the choice of the sources to be estimated as local parameters (see, e.g. Fig. 2 of Lambert et al. 2008).

In the present paper, we only deal with the reference sources, and this explains the bias variations between EOP solutions. However, no significant variations in $t$ or $t^{2}$ are found. Such a result was expected and does not contradict the other works mentioned above, since we strictly use the same split between local and global source coordinates in all of our solutions. Finally, no effect is detectable in station coordinate and velocity catalogues, indicating that the choice of reference radio sources, when these radio sources constitute a reasonably stable reference frame, does not influence the realization of the terrestrial reference frame.

\section{Concluding remarks}

This study investigates various reference frames made of different subsets of radio sources. They contain 196, 200, 262, and 269 sources, respectively. They were selected after a statistical analysis of radio source coordinate time series, and were compared to the frames defined by the 212 ICRF defining sources and by the 247 stable sources of Feissel-Vernier et al. (2006) in terms of axis time stability. The frames made of 196 and 262 sources appear to be $20 \%$ more stable than the frame defined by the 247 stable sources of Feissel-Vernier et al., and $40 \%$ more stable than 
the frames defined by the 212 ICRF defining sources. Applying a NNR condition to our selected sources for a global VLBI analysis permits us to obtain astrometric catalogues non rotated (or by negligible amount) with respect to the ICRF-Ext.2. No drastic consequences for EOP and terrestrial frame determination appear.

These stable frames are not unique and are mainly the result of selection effects caused by the observational history of sources. Indeed, sources that are not often observed are unlikely to be selected since one simply cannot determine whether they have a stable position. However, since the VLBI data set used in this study is representative of the whole observational data base (which represents about 4000 diurnal sessions), using a different, but close, observational data set to produce coordinate time series would lead to a source selection that would be close to ours. We therefore recommend that such a selection algorithm be used in the generation of the next ICRF, along with other selection criteria based on compactness or observability.

\section{References}

Feissel, M., \& Gontier, A.-M. 2000, in International VLBI Service for Geodesy and Astrometry (IVS), General Meeting, NASA/CP-2000-209893, ed. N. R. Vandenberg, \& K. D. Baver, 280

Feissel-Vernier, M. 2003, A\&A, 403, 105

Feissel-Vernier, M., Ray, J., Altamimi, Z., et al. 2004, in International VLBI Service for Geodesy and Astrometry (IVS) General Meeting, ed. N. R. Vandenberg, \& K. D. Baver, NASA/CP-2004-212255, 22

Feissel-Vernier, M., Ma, C., Gontier, A.-M., \& Barache, C. 2006, A\&A, 452, 1107
Fey, A. L., \& Charlot, P. 2000, ApJS, 128, 17

Fey, A. L., Ma, C., Arias, E. F., et al. 2004, AJ, 127, 3587

Gontier, A.-M., Le Bail, K., Feissel, M., \& Eubanks, T. M. 2001, A\&A, 375, 661

Gontier, A.-M., \& Lambert, S. B. 2008, in Proc. Journées 2007 systèmes de référence spatio-temporels, Observatoire de Paris, ed. N. Capitaine, 42

IERS 1996, International Earth Rotation Service Annual Report 1995 (Observatoire de Paris), II-19

Lambert, S. B., Dehant, V., \& Gontier, A.-M. 2008, A\&A, 481, 535

Ma, C., Arias, E. F., Eubanks, T. M., et al. 1998, AJ, 116, 516

MacMillan, D. S., \& Cohen, S. 2004, in International VLBI Service for Geodesy and Astrometry (IVS) 2004 General Meeting Proceedings, NASA/CP-2004212255, ed N. R. Vandenberg, \& K. D. Baver, 491

MacMillan, D. S., \& Ma, C. 2007, J. Geod., 81, 443

Malkin, Z. 2004, in international VLBI Service for Geodesy and Astrometry (IVS), General Meeting, NASA/CP-2004-212255, ed. N. R. Vandenberg, \& K. D. Baver, 47

Mathews, P. M., Herring, T. A., \& Buffett, B. A. 2002, J. Geophys. Res., 107(B4), 10.1029/2001JB000390

Mignard, F., \& Morando, B. 1990, in Proc. Colloque André Danjon, Journées 1990 systèmes de référence spatio-temporels, Observatoire de Paris, ed. N. Capitaine, \& S. Débarbat, 151

Niell, A. E. 1996, J. Geophys. Res., 101(B2), 3227

Petrov, L. 2005, in European VLBI for Geodesy and Astrometry (EVGA) 17th Working Meeting Proceedings, ed. M. Vennebusch, \& A. Nothnagel, 113

Schlüter, W., \& Behrend, D. 2007, J. Geod., 81, 479

Sokolova, J. R. 2008, in Proc. Journées 2007 systèmes de référence spatiotemporels, Observatoire de Paris, ed. N. Capitaine, 24

Sokolova, J. R., \& Malkin, Z. 2007, A\&A, 474, 665

Titov, O., \& Tregoning, P. 2004, in international VLBI Service for Geodesy and Astrometry (IVS), General Meeting Proceedings, NASA/CP-2004-212255, ed. N. R. Vandenberg, \& K. D. Baver, 496

Titov, O., \& Tregoning, P. 2005, J. Geod., 79, 196

Titov, O. 2007, J. Geod., 81, 455 